\title{
Éditorial
}

\section{Rencontre intergouvernementale}

La Septième Réunion du Groupe de Travail Technique Intergouvernemental sur les Ressources Zoogénétiques pour l'Alimentation et l'Agriculture s'est tenue à Rome en Octobre 2012. ${ }^{1}$ Comme il a été dit dans l'éditorial du volume 51, une des principales tâches que le Groupe de Travail a dû confronter a été celle d'examiner les progrès réalisés dans la mise en ouvre du Plan d'Action Mondial pour les Ressources Zoogénétiques. ${ }^{2}$ Le Groupe de Travail a fait bon accueil au substantiel progrès réalisé, en admettant cependant qu'il existe encore des lacunes à combler, notamment en ce qui concerne l'amélioration de la collaboration et du financement des activités. Pour plus de détails, veuillez consulter le document Synthesis progress report on the implementation of the Global Plan of Action for Animal Genetic Resources - 2012 (Rapport intérimaire de synthèse sur la mise en œuvre du Plan d'Action Mondial pour les Ressources Zoogénétiques - 2012), ${ }^{3}$ qui a été préparé pour la réunion du Groupe de Travail.

L'information présentée dans le Rapport intérimaire de synthèse a aussi été utilisée pour préparer un ensemble de trois affiches :

1. Strategic priorities of the Global Plan of Action for Animal Genetic Resources ${ }^{4}$ (Priorités stratégiques du Plan d'Action Mondial pour les Ressources Zoogénétiques; vue d'ensemble des priorités stratégiques du Plan d'Action Mondial aux échelles nationale, régionale et mondiale);

2. Status of implementation of the Global Plan of Action for Animal Genetic Resources ${ }^{5}$ (État de mise en œuvre du Plan d'Action Mondial pour les Ressources Zoogénétiques; indicateurs pour chaque priorité stratégique aux niveaux national, régional et mondial); et

3. Colour scheme expressing levels of implementation of the Global Plan of Action for Animal Genetic Resources $^{6}$ (Échelle de couleur représentant les degrés de mise en œuvre du Plan d'Action Mondial pour les Ressources Zoogénétiques; échelle de couleur utilisée pour illustrer les indicateurs).

La préparation et planification d'une mise à jour de L'État des Ressources Zoogénétiques pour l'Alimentation et l'Agriculture dans le Monde étaient aussi à l'agenda du Groupe de Travail. Il a été prévu de présenter un deuxième rapport à la Commission des Ressources

\footnotetext{
http://www.fao.org/docrep/meeting/027/mf227f.pdf

${ }^{2} \mathrm{http} / / /$ www.fao.org/docrep/010/a1404f/a1404f00.htm

${ }^{3} \mathrm{http}: / /$ www.fao.org/docrep/meeting/026/me636e.pdf

${ }^{4} \mathrm{ftp}$ :/DADnet:Mobile45@ext-ftp.fao.org/ag/reserved/dad-net/GPA.pdf

${ }^{5} \mathrm{ftp} / / /$ DADnet:Mobile45@ext-ftp.fao.org/ag/reserved/dad-net/Indicator.pdf

${ }^{6} \mathrm{ftp} / / /$ DADnet:Mobile45@ext-ftp.fao.org/ag/reserved/dad-net/Traffic light.pdf
}

Génétiques pour l'Alimentation et l'Agriculture en 2017, dix ans après la publication du premier rapport. Toutefois, en raison de la lourde charge de travail programmée pour la Seizième Réunion Ordinaire de la Commission en 2017, il a été demandé au Groupe de Travail de prendre en considération la possibilité d'avancer la préparation du deuxième rapport de deux ans, c'est-à-dire de conseiller à la Commission qu'elle sollicite à la FAO de présenter le rapport lors de la Quinzième Réunion Ordinaire de la Commission en 2015, ce qui impliquerait qu'une ébauche serait soumise à l'examen du Groupe de Travail vers la fin de l'année 2014. Le Groupe de Travail a recommandé à la Commission d'accepter cette programmation. Ce volume de Ressources Génétiques Animales a été mis sous presse avant la réunion de la Commission du mois d'avril, au cours de laquelle la recommandation du Groupe de Travail devait être examinée.

Qu'il soit présenté en 2015 ou ultérieurement, le deuxième rapport constituera une mise à jour du premier rapport d'autant plus qu'il présentera les changements survenus entre-temps pour ce qui est de l'état des ressources zoogénétiques et leur gestion et les derniers développements scientifiques dans les domaines pertinents. La charge de travail que représente la rédaction des rapports pour les pays serait réduite à un minimum, l'information sur l'état de la gestion des ressources zoogénétiques à l'échelon national étant obtenue par le biais de questionnaires spécifiques plutôt qu'en demandant aux pays d'élaborer des rapports détaillés du genre de ceux préparés pour le premier rapport.

Les points suivants étaient aussi à l'agenda du Groupe de Travail: une révision de la mise en œuvre de la Stratégie de Financement pour la mise en application du Plan d'Action Mondial pour les Ressources Zoogénétiques (la rencontre a coïncidé avec l'annonce de la première série de projets sélectionnés pour recevoir le soutien de la Stratégie de Financement) ${ }^{7}$ les rôles des petits éleveurs dans la conservation et l'utilisation durable des ressources zoogénétiques; objectifs et indicateurs pour les ressources zoogénétiques; situation et tendances en ce qui concerne les micro-organismes intervenant dans la digestion chez le ruminant; et une révision des résultats de la Première Réunion du Groupe de Travail de la Commission sur l'Accès et le Partage des Avantages.

\section{Trente ans de la Commission}

En plus d'être une année potentiellement occupée pour la communauté internationale des ressources zoogénétiques,

\footnotetext{
${ }^{7} \mathrm{http}: / /$ www.fao.org/ag/againfo/programmes/fr/genetics/Funding strategy.html
} 
l'année 2013 marque le trentième anniversaire de la Commission. Fondée en 1983 sous le nom de Commission des Ressources Phytogénétiques pour l'Alimentation et l'Agriculture, la Commission peut regarder en arrière sur une longue série de réussites, parmi lesquelles la négociation du Traité International sur les Ressources Phytogénétiques pour l'Alimentation et l'Agriculture et la préparation de Rapports sur l'État Mondial et Plans d'Action Mondiaux dans les domaines des ressources phytogénétiques et zoogénétiques. Initialement focalisé sur les cultures, le travail de la Commission s'est étendu par la suite aux animaux (l'élevage), puis à la sylviculture, aux ressources génétiques aquatiques et enfin aux invertébrés et microorganismes. L'établissement de liens entre les différents secteurs des ressources génétiques ainsi que leur rôle dans la prestation de services aux écosystèmes retiennent de plus en plus l'attention de la Commission, en particulier en vue de la préparation projetée d'un rapport sur L'État de la Biodiversité pour l'Alimentation et l'Agriculture.

\section{Archives du journal}

Les lecteurs seront peut-être curieux d'apprendre que tous les numéros antérieurs de Ressources Génétiques Animales sont disponibles sur le site web de Cambridge University Press, ${ }^{8}$ qui est aussi équipé d'un puissant moteur de recherche. 